\title{
Paraganglioma of Urinary Bladder Managed by Laparoscopic Partial Cystectomy in Conjunction with Flexible Cystoscopy: A Case Report
}

\author{
Hossam S. El-Tholoth, MD, Sami Al Rasheedi, MBBS, Faris Alharbi, MBBS, \\ Waleed Alshammari, MBBS, Tarek Alzahrani, SUB, and Ahmed Al Zahrani, SUB
}

\begin{abstract}
Background: Paraganglioma of the urinary bladder (PUB) is exceedingly rare, accounting for $<0.1 \%$ of all urinary bladder tumors. Various challenging treatment options are available.

Case Presentation: A 67-year-old female presented with malignant hypertension on four medications for which investigation was done. An observation of having functioning PUB was noted. She was admitted and laparoscopic partial cystectomy was done with the guidance of flexible cystoscopy. She had a smooth postoperative course and was discharged home, then catheter was removed after cystogram. Histopathology confirmed the diagnosis of a bladder paraganglioma. Finally, during the last follow-up, the patient was asymptomatic with controlled blood pressure and normalized catecholamine levels with no evidence of recurrence.

Conclusion: PUB is an exceedingly rare tumor that can be managed with minimally invasive techniques such as laparoscopic partial cystectomy with cystoscopy guidance.
\end{abstract}

Keywords: paraganglioma of urinary bladder, laparoscopic partial cystectomy, cystoscopy

\section{Introduction}

$\mathbf{P}$ ARAGANGLIOMAS OF THE URINARY BLADDER (PUB) is remarkably rare, accounting for $<0.1 \%$ of all urinary bladder tumors.

\section{Case Presentation}

A 67-year-old female presented with malignant hypertension on four medications for which investigation by an endocrinologist showed positive metaiodobenzylguanidine scan (MIBG), elevated catecholamines levels, and CT with contrast showed intravesical tumor with a tentative diagnosis of bladder paraganglioma of size $3.5 \times 3 \mathrm{~cm}$ as seen in Figure 1 and Figure 2.

She was admitted and was prepared for surgery. Laparoscopic partial cystectomy was conducted (she was placed in low lithotomy position and laparoscopic procedure was started by mobilizing the bladder in combination with flexible cystoscopy that showed a mucosal bulge located in anterior bladder wall with a wide base near the bladder neck. The light of the endoscope guided the start of resection with a safety margin and then we excised the mass completely with a good safety margin as shown in Figure 3, and bladder was closed in one layer laparoscopically. During surgery, she developed one attack of hypertension and was managed by anesthesia with no intraoperative or immediate postoperative complications. She had a smooth postoperative course and was discharged home, then the catheter was removed after cystogram. Histopathology confirmed the diagnosis of bladder paraganglioma (Fig. 4). As well, a follow-up at 1 year postoperation, the patient was asymptomatic with controlled blood pressure and normalized catecholamines level with no evidence of recurrence.

\section{Conclusion}

PUB is an exceedingly rare tumor that can be managed with minimally invasive techniques such as laparoscopic partial cystectomy with cystoscopy guidance.

Department of Urology, Prince Sultan Military Medical City, Riyadh, Saudi Arabia.

(C) Hossam S. El-Tholoth et al. 2018; Published by Mary Ann Liebert, Inc. This Open Access article is distributed under the terms of the Creative Commons License (http://creativecommons.org/licenses/by/4.0), which permits unrestricted use, distribution, and reproduction in any medium, provided the original work is properly cited. 


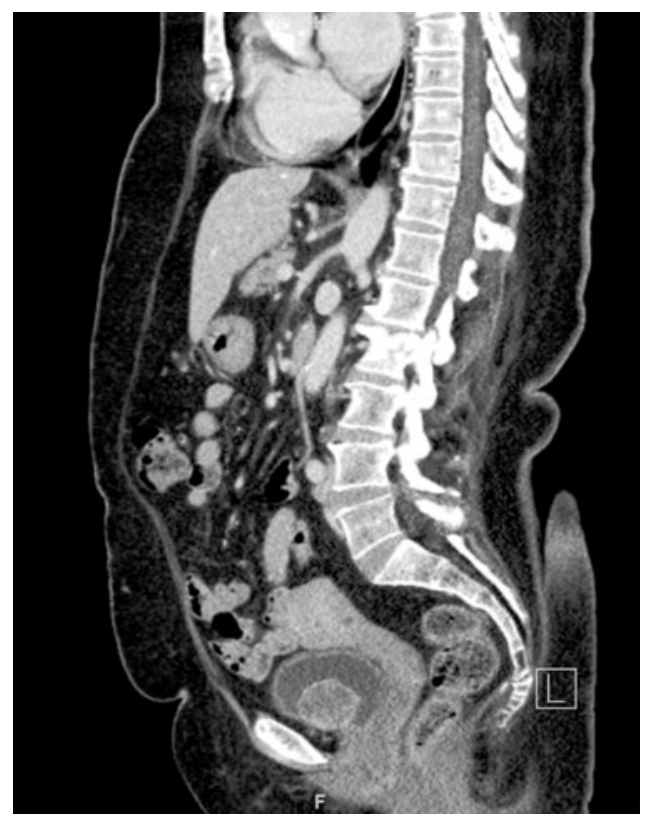

FIG. 1. Sagittal reconstructed CT scan image showing bladder mass.

\section{Discussion}

PUB is rare $<1 \%$ of bladder tumors and $6 \%$ of all paragangliomas. Out of these PUB, only $10 \%$ show malignant behavior. There are no specific sites inside the urinary bladder more vulnerable to PUB. However, it is commonly reported at the lateral wall, posterior wall, and trigone. PUB can



FIG. 2. MIBG scintigraphy_sagittal view showing bladder mass. MIBG, metaiodobenzylguanidine scan.

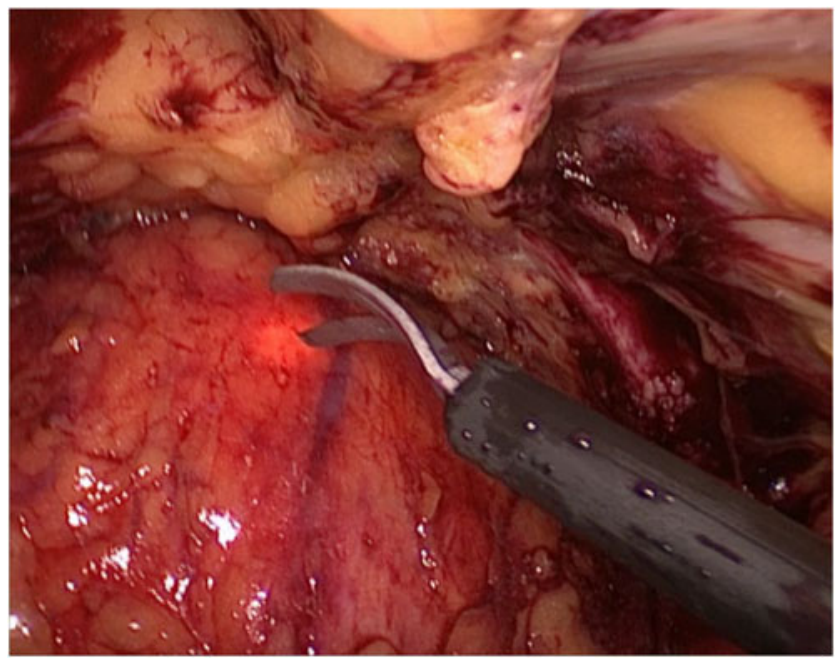

FIG. 3. Laparoscopic view to start resection over guidance of cystoscopy light.

be misdiagnosed with other urothelial bladder tumors. Most of the reported cases are younger than those of urothelial carcinoma with a median age of 43 years. ${ }^{1,2}$ In our case study, the patient is a 67-year-old coping with the median age of other urothelial tumors. The clinical presentation of functioning paraganglinoma is manifested by a headache and uncontrolled hypertension despite medications. The use of MIBG and elevated urinary catecholamine levels help the tentative diagnosis of the case. Immunohistochemistry and characteristic histologic features are the cornerstones for confirmative diagnosis with high index of clinical suspicion. ${ }^{2}$ There are various treatment options for PUB, including transurethral resection, partial cystectomy, and radical cystectomy. ${ }^{3}$ Because the paraganglionoma of the urinary bladder in our case was preoperatively known to be functioning, a large size, and located in the anterior bladder wall, laparoscopic partial cystectomy was the preferred option of treatment, as intraoperatively uncontrolled hypertension may

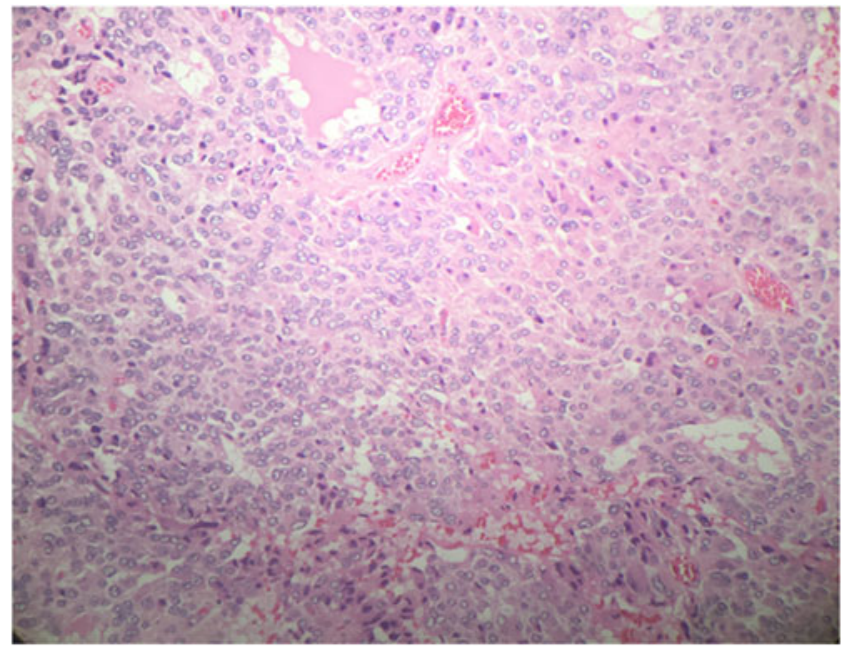

FIG. 4. Histopathology slide of the lesion confirming the diagnosis of bladder paraganglioma. 
be disastrous in such cases. To the best of our knowledge, the only laparoscopically assisted partial cystectomy for a known preoperative functional paraganglinoma that was effectively treated without sequels under cystoscopic guidance was previously reported by Gofrit et al. ${ }^{4}$ for open partial cystectomy.

\section{Disclosure Statement}

No competing financial interests exist.

\section{References}

1. Lin O, Cardillo M, Dalbagni G, et al. Nested variant of urothelial carcinoma: A clinicopathological and immunohistochemical study of 12 cases. Mod Pathol 2003;16:1289-1298.

2. Thrasher JB, Rajan RR, Perez LM, et al. Pheochromocytoma of urinary bladder: Contemporary method of diagnosis and treatment options. Urology 1993;41:435-439.

3. Purnell S, Sidana A, Maruf M, et al. Genitourinary paraganglioma: Demographic, pathologic, and clinical characteristics in the surveillance, epidemiology, and end results database (2000-2012). Urol Oncol 2017;35:457.

4. Gofrit ON, Shapiro A, Katz R, et al. Cystoscopic-assisted partial cystectomy: Description of technique and results. Res Rep Urol 2014;6:139-143.
Address correspondence to: Hossam S. El-Tholoth, MD Department of Urology

Prince Sultan Military Medical City No. 7889, Makkah Almokarama Branch Road

Riyadh 11159

Saudi Arabia

Email: urologist_hossam@yahoo.com

Cite this article as: El-Tholoth HS, Al Rasheedi S, Alharbi F, Alshammari W, Alzahrani T, Al Zahrani A (2018) Paraganglioma of urinary bladder managed by laparoscopic partial cystectomy in conjunction with flexible cystoscopy: a case report, Journal of Endourology Case Reports 4:1, 15-17, DOI: 10.1089/ cren.2017.0132. 